\title{
Characterization of Coriander Sativum Mediated Silver Nanoparticles and Evaluation of Its Antimicrobial and Wound Healing Activity
}

\author{
M.Balamurugan, Sami Nathan R., \\ M.Tech Department of Textile Technology, Jaya Engineering College, Thiruninravur. \\ Professor and Department Head Department of Textile Technology, Jaya Engineering College, Thiruninravur
}

\begin{abstract}
To meet the increasing demands for commercial nanoparticles new eco-friendly "green" methods of synthesis are being discovered. Plant mediated synthesis of nanoparticles offers single step, easy extracellular synthesis of nanoparticles. We report the synthesis of antibacterial silver nanoparticles using leaf broth of medicinal herb Coriandersativum. The completion of the formation of silver nanoparticles was monitored by $U V$-visible spectroscopy. The particle size of the synthesized silver nanoparticles was found by XRD. The silver nanoparticles stabilized by C. stadium leaf extract were found to have enhanced antimicrobial activity against well-known pathogenic only available strains.
\end{abstract}

\section{Introduction}

Nanotechnology is emerging as a rapidly growing field with its application in science and technology for the purpose of manufacturing new materials at the Nano scale level. In the present scenario, Nano scale materials have emerged up as novel antimicrobial agents owing to their high surface area to volume ratio and the unique chemical and physical properties [1],[2]. Silver has come up but silver nanoparticles have proved to be most effective as it has good antimicrobial efficacy against bacteria, viruses and other eukaryotic microorganisms [3]. Several physical, chemical and biological synthesis methods have been developed to enhance the performance of nanoparticles displaying improved properties with the aim to have a better control over the particle size, distribution and morphology [4]. Synthesis of nanoparticles to have a better control over particles size, distribution, morphology, purity, quantity and quality, by employing environment friendly economical processes has always been a challenge for the researchers [5]. The current investigation supports that use of silver ion or metallic silver as well as silver nanoparticles can be exploited in medicine for burn treatment, dental materials, coating stainless steel materials, textile fabrics, water treatment, sunscreen lotions, etc. and possess low toxicity to human cells, high thermal stability and low volatility [ 6]. Nanotechnology is now creating a growing sense of excitement in the life sciences especially biomedical devices and Biotechnology [7].Wound healing activity is the body's natural process of regenerating dermal and epidermal tissues. It is the process whereby body restores the injured part to as near its normal condition as possible. Though wound healing takes place naturally own its own, some of the complications like sepsis, distribution of tissue and skin layer, maggot's formation and extension of infection to adjacent and interior organs occur in major cases. To prevent extensive loss and damage to the tissue, skin grafting biological dressing was developed [8].Dressings play a major role in the management of wounds "In recent times, the development of resistant strains of pathogens has become a major problem and the newly designed wound dressings has provided a major breakthrough for the treatment of infection. Silver is used in different kinds of formulations like surface coatings agents, wound dressings [9]. It is confirmed that silver nanoparticles are capable of rendering high antifungal efficacy and hence has a great potential in the preparation of drugs used against fungal diseases [16].

\section{Materials And Methods}

Collection and drying of plant materials mature leaves of coriandrumsativum were collected from Rajapalayam, Tamil Nadu. The leaves of c.sativum were washed thoroughly three times with water and once with distilled water. The plant materials were air dried and powdered. The powdered samples were hermetically sealed in separate polythene bags until the time of extraction.

Preparation of plant Extract $10 \mathrm{~g}$ of powdered leaves were extracted successively with $100 \mathrm{ml}$ of methanol at $40-50^{\circ} \mathrm{C}$ in soxhelet extractor until the extract was clear. The extracts were evaporated to dryness and the resulting pasty form extracts were stored in a refrigerator at $4^{\circ} \mathrm{C}$ for future use [10],[11].

$\mathrm{UV}-\mathrm{Vis}$ spectral analysis

After incubation, the color change in leaf and fruit extract was compared with control and sample. Synthesized silver nanoparticles was confirmed by sampling the aqueous component and the absorption maximum was scanned by UV Vis spectrometer at the wavelength $400-450 \mathrm{~nm}$ on per kin-Elmer Lambda 25 
spectrophotometer. Based on the absorbance value and peak it shows that which plant extract has the highest ability to synthesize nanoparticles.

Test Microorganisms

Nine pathogenic bacteria, viz., staphylococcus aurous, Escherichia coli, klebsiella pneumonia, and proteus vulgarisand pseudomonas aeruginosa species of bacteria were used during the present study and were obtained from Kalasalingam University. The cultures were sub-cultured and maintained on nutrient agar slants and stored at $4^{\circ} \mathrm{C}$.

Antibacterial Activity

The antibacterial activity test was taken out .by using prepare instruments. It was then incubated at $37^{\circ} \mathrm{C}$ for 24hrs. The test were examined for antibacterial purpose.

Particle size analysis

It was performed at Kalasalingam University, srivilliputhur. The particle size distribution of silver nanoparticles was evaluated using dynamic light scattering (DLS) measurements conducted with a malvern zetasizer nano series compact scattering spectrometer. Data obtained were analyzed using zetasizer software Microorganisms for fabric. Wound healing activity was carried out using fabric aid for performing wound healing activity. Fabric wasprepared.

\section{Results And Discussion}

The green synthesis of silver nanoparticles through plant extracts were carried out. It is well known that silver nanoparticles exhibit yellowish - brown color in aqueous solution due to excitation of surface Plasmon vibrations in silver nanoparticles [13]. The appearances of yellowish-brown color in the reaction vessels suggest the formation of silver nanoparticles (SNPs)[14]. Silver nitrate is used as precursor and good antibacterial activity as silver has distinctive properties such as good conductivity, catalytic and chemical stability. The time duration of change in color varies from plant to plant. The synthesis of SNPs had been confirmed by measuring the UV-Vis spectrum of the reaction media. The UV-Vis spectrum of colloidal solutions of SNPs synthesized from, Coriandrum sativum have absorbance peaks at 400 to $450 \mathrm{~nm}$ at $24 \mathrm{hrs}$.' and 48 hrs.' (Fig1 and 2). Toxicity studies on pathogen opens a door for nanotechnology applications in medicine. c.sativum extract, Staphylococcus aurous produce the maximum zone of inhibition (28mm) and the minimum zone of inhibition was produced by Klebsiella pneumonia $(17 \mathrm{~mm})$ (Table 1). The coriander sample was freeze dried, sonicated with distilled water, small drop of this sample was placed on glass slide allowed to dry 15 revealed that, there were two peaks in the beginning, and they continue to exist even up to 24 hrs.' with slight increase in intensity. This indicates

Non-uniform distribution of size in the beginning of the reaction. A thin layer of platinum was coated to make the samples conductive Joel JSM-6840 LV SEM machine was operated at a vacuum of the order of 105 torr. The accelerating voltage of the microscope was kept in the range 10-20 kv. Compositional analysis carried on the sample. The sample was sent to Kalasalingam University, srivilliputhur (Fig 4).

Table 1: Antibacterial activity of Coriander sample against bacterial pathogens

\begin{tabular}{lllll}
\hline S.No & \multicolumn{1}{c}{ Organism } & \multicolumn{3}{c}{$\begin{array}{c}\text { Concentration of extract and zone } \\
\text { of inhibition(mm) }\end{array}$} \\
\cline { 3 - 5 } & & Plant extract & $\begin{array}{l}\text { Silver } \\
\text { nitrate }\end{array}$ & Sample \\
\hline 1 & Escherichia coli & - & $12 \mathrm{~mm}$ & $21 \mathrm{~mm}$ \\
2 & Klebsiella pneumonia & - & $11 \mathrm{~mm}$ & $17 \mathrm{~mm}$ \\
3 & Pseudomonas aeruginosa & - & $11 \mathrm{~mm}$ & $27 \mathrm{~mm}$ \\
4 & Proteus valgaris & - & $14 \mathrm{~mm}$ & $18 \mathrm{~mm}$ \\
5 & Staphylococcus aureus & - & $16 \mathrm{~mm}$ & $28 \mathrm{~mm}$ \\
\hline
\end{tabular}

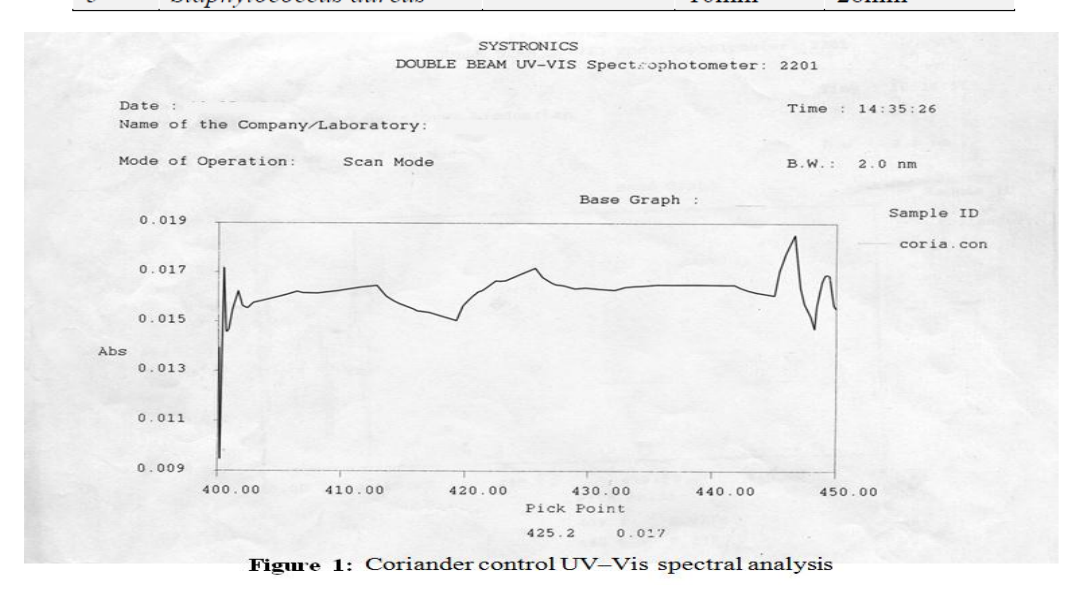

www.iosrjournals.org 

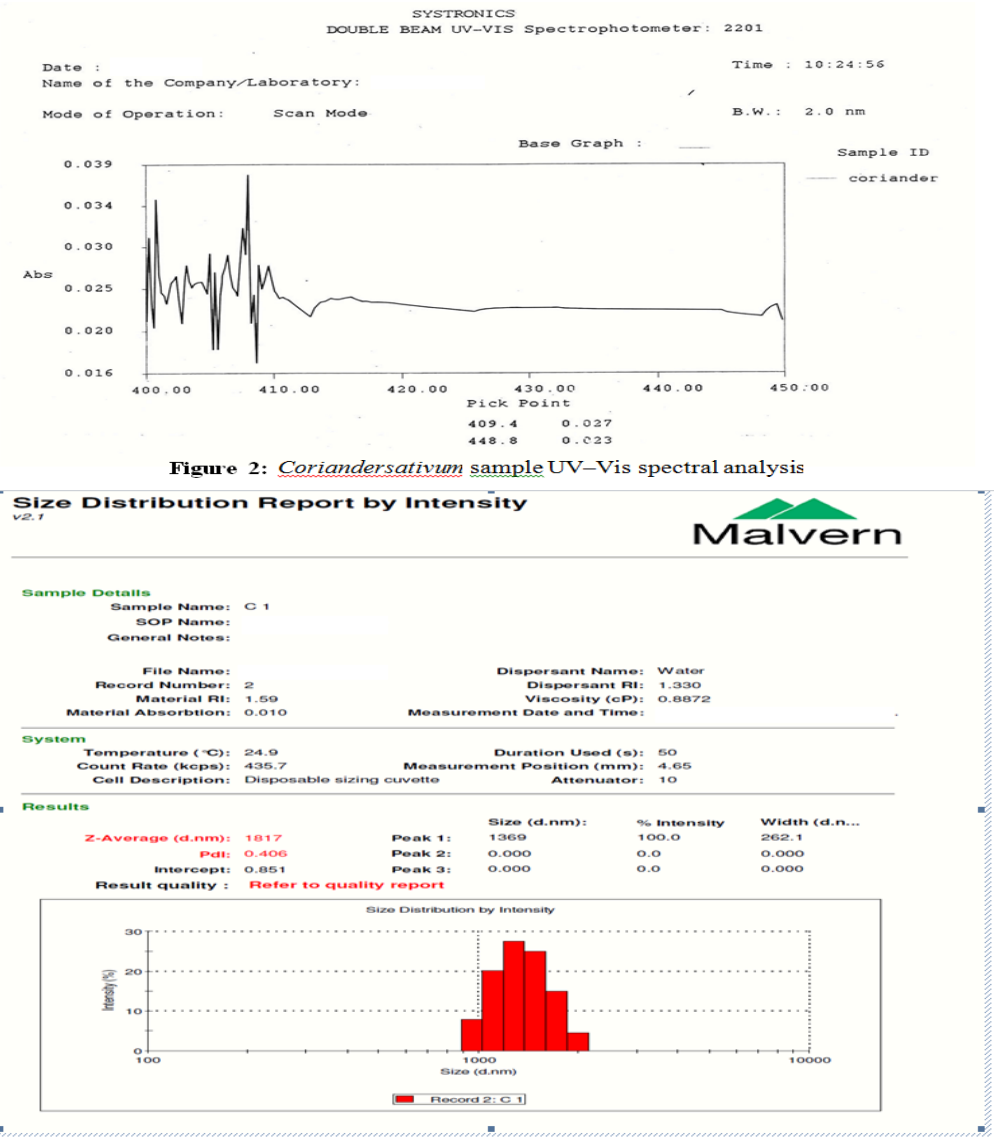

Figure 3: Size distribution report of coriander sample

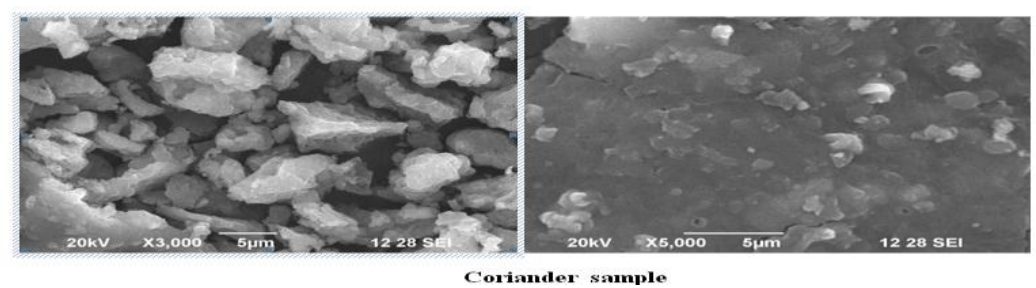

IV. Conclusion

The present study included the bio-reduction of silver ions through medicinal plants extracts and testing for their antimicrobial activity. The aqueous silver ions exposed to the extracts, the synthesis of silver nanoparticles were confirmed by the change of color of plant extracts. These environmentally benign silver nanoparticles were further confirmed by using UV-Vis spectroscopy and SEM analysis. It is confirmed that silver nanoparticles are capable of rendering high antibacterial efficacy and hence has a great potential in the preparation of drugs used against bacterial diseases.

\section{References}

[1]. Morones JR and Elechiguerra JL, Alejandra Camacho, Katherine Holt, Juan B Kouri, Jose Tapia Ramirez and Miguel Jose Yacaman. Nanotechnology, 2005 16: 23-46.

[2]. Kim JS, Kuk E, Yu KN, Kim JH, Park SJ, Lee HJ, Kim SH and Park YK. NanomedNanotechnolBiolMed, 2007,3(1):95-101

[3]. Gong P, Li, H, He, X, Wang, K, Hu, J, Zhang S and Yang X. Nanotechnology 2007, 18(28): 604-611

[4]. Shankar S. S., Ahmad, A. and Sastry M. Geranium leaf assisted biosynthesis of silver nanoparticles. Biotechnology Prog, 2003, 19:1627-1631.

[5]. PrasadT.N.V.K.V., Elumalai E.K., Khateeja S. Evaluation of the antimicrobial efficacy of phytogenic silver nanoparticles. Asian pacific journal of tropical Biomedicine,2011:s82-s85.

[6]. Duran N, Marcarto, PD, De Souza, GIH., Alves, OL and Esposito E. J Biomed Nanotechnol2007, 3( 2): $203-208$.

[7]. Prabhu N, Divya TR, Yamuna G. Digest J NanomaterBiostruct, 2010,5: 185-189.

[8]. Duran N, Marcato P, DAlves O L,Souza G.I.H.Mechanistic aspects of biosynthesis of silver nano particles by several Fusariumoxysporumstrains,Nanobiotechnology,2005,3:8-14. 
[9]. Anjaiah A,Harogopal V, Raga Vander KBP and Chandrasekhar EL.Effect of full thickness skin grafts and mesh skin grafts on granulating wounds in dogs, Experimental study,2001,30:92-94

[10]. Chessbrough M. Medical laboratory manual for Tropical countries, Linacre House, Jordan Hill, Oxford 2000

[11]. Evans WC. Trease and Evan's Pharmacognosy. 5th edition, Harcourt Brace and Company: 2002, 336.

[12]. Singh RP, Magesh S, And Rakkiyappan C. Ginger (Zingiberofficanale) Root extract: A source of silver nanoparticles and their applications.Int.Journal of Bio-Eng. Science and tech., 2011, 2(3):75-80.

[13]. Thirumurgan A, Tomy NA, Jai Ganesh R and Gobi Krishnan S. De Phar Chem., 2010, 2: 279-284

[14]. Shankar SS, Rai A, Ahmad A and SastryMJ. J Collid Interface Sci, 275: 2004.496-502.

[15]. Avilajerley A, and Anita marget, A. Mycosynthesis of silver nanoparticles and antibacterial effects in wound dressing. International, j, of Pharm. Sciences and researches. 2012, 3(6): 1698-1704. 16. Vasantharaj S, Sathiyavimal S, and Hemashenpagam N: Antimicrobial Activity of Silver Nano particle Synthesized by Using Costusigneus.Res. J. Pharm. Bio.andChe. Sci 2013 , 733-738. 